\title{
Association of serum Fetuin-B with insulin resistance and pre- diabetes in young Chinese women: evidence from a cross- sectional study and effect of liraglutide
}

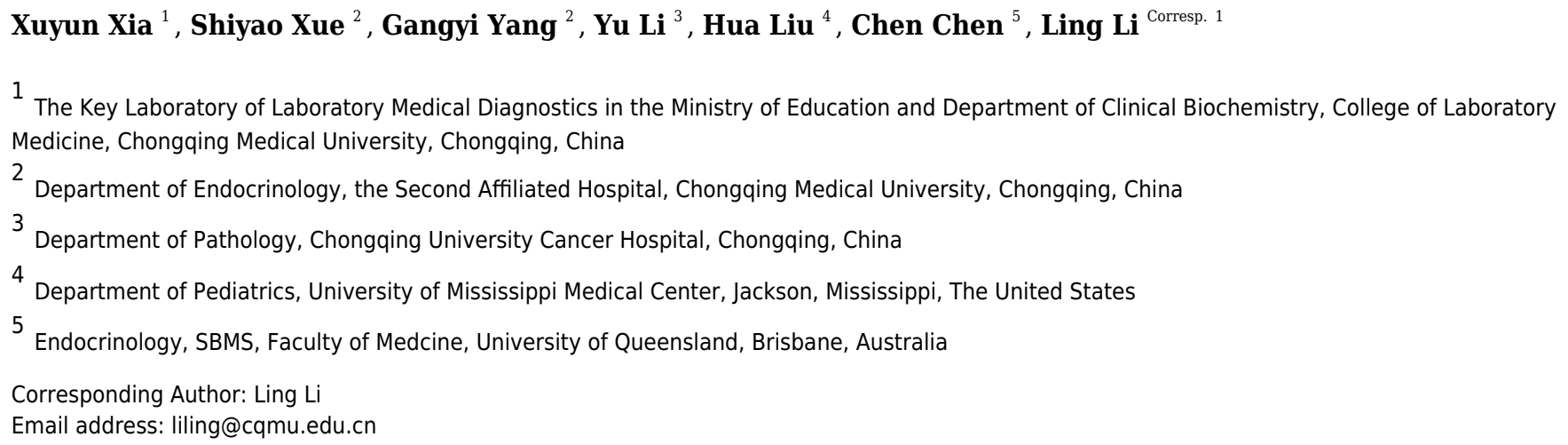

Background and Aims. Fetuin-B has been reported to be involved in glucose and lipid metabolism and associated with the occurrence of diabetes. The main purpose of this study is to explore the changes of circulating Fetuin- $B$ in young wome $n$ with pre-diabetes and to analyze the relationship between Fetuin- $B$ and the occurrence and development of IR.

Methods. A total of 304 women were enrolled in this study and subjected to both OGTT and EHC. A subgroup of 26 overweight/obese women was treated with Lira for 24 weeks. Serum Fetuin-B concentrations were measured by ELISA.

Results. In IGT and IR-NG groups, serum Fetuin-B levels were higher than those in the NGT group. The serum Fetuin-B levels in the IGT group were higher than those in the IR-NG group. Serum Fetuin-B was positively correlated with BMI, WHR, 2 h-BG, FIns, HbA1c, and HOMA2-IR, but negatively correlated with the M-value in all study populations. Multiple stepwise regression analysis showed that the M-value was independently and inversely associated with serum Fetuin-B. Logistic regression analysis showed that serum Fetuin-B was independently associated with IGT and significantly increased the risk of IGT. During the OGTT, serum Fetuin-B increased significantly in the NGT group, but there were no significant changes in other groups. During the EHC, serum Fetuin-B increased in the IGT group, but there was no change in other groups. After Lira intervention, serum Fetuin-B decreased significantly in IGT women.

Conclusions. Serum Fetuin-B levels are elevated in young women with IR or IGT and may be associated with IR. 
1 Association of serum Fetuin-B with insulin resistance and pre-diabetes in young Chinese

2

3

$4{ }^{1}$ The Key Laboratory of Laboratory Medical Diagnostics in the Ministry of Education and

5

6

7

8 Chongqing, China

9

10

11

12 
Background and Aims. Fetuin-B has been reported to be involved in glucose and lipid metabolism and associated with the occurrence of diabetes. The main purpose of this study is to explore the changes of circulating Fetuin-B in young women with pre-diabetes and to analyze the relationship between Fetuin-B and the occurrence and development of IR.

Methods. A total of 304 women were enrolled in this study and subjected to both OGTT and EHC. A subgroup of 26 overweight/obese women was treated with Lira for 24 weeks. Serum Fetuin-B concentrations were measured by ELISA.

Results. In IGT and IR-NG groups, serum Fetuin-B levels were higher than those in the NGT group. The serum Fetuin-B levels in the IGT group were higher than those in the IR-NG group. Serum Fetuin-B was positively correlated with BMI, WHR, 2 h-BG, FIns, HbA1c, and HOMA2IR, but negatively correlated with the M-value in all study populations. Multiple stepwise regression analysis showed that the M-value was independently and inversely associated with serum Fetuin-B. Logistic regression analysis showed that serum Fetuin-B was independently associated with IGT and significantly increased the risk of IGT. During the OGTT, serum Fetuin-B increased significantly in the NGT group, but there were no significant changes in other groups. During the EHC, serum Fetuin-B increased in the IGT group, but there was no change in other groups. After Lira intervention, serum Fetuin-B decreased significantly in IGT women. Conclusions. Serum Fetuin-B levels are elevated in young women with IR or IGT and may be associated with IR.

Key Words: Fetuin-B; pre-diabetes; insulin resistance; Liraglutide.

Abbreviations: IR, insulin resistance; IGT, impaired glucose tolerance; ELISA, enzyme-linked 
43 immunosorbent assay; OGTT, oral glucose tolerance test; EHC, Euglycemic- hyperinsulinemic

44 clamp; Lira, liraglutide; BMI, body mass index; WHR, waist-to-hip ratio; 2 h-BG, 2-hour blood

45 glucose post-glucose load; FIns, fasting insulin; HOMA2-IR, homeostasis model assessment of 46 insulin resistance.

47

48 Short title: Fetuin-B is associated with pre-diabetes

49

Clinical Trial Registration Number: ChiCTR-OCC-11001422

Introduction

61 Type 2 diabetes mellitus (T2DM) is a metabolic disease characterized by relative insulin deficiency caused by impaired islet function and target organ insulin resistance (IR). As the main epidemic area of diabetes in Asia, China has become the country with the largest number of 
64 diabetic patients in the world (Chatterjee, et al. 2017). Traditionally, T2DM has been considered

65 a chronic disease related to the elderly, but with rapid economic and social development in recent

66 years, changes in lifestyle and diet structure have resulted in increasing rates of T2DM and

67 obesity in adolescents (Arcidiacono, et al. 2020; Lascar et al. 2018). According to a 2010

epidemiological survey in China, the rate of diabetes in China is $4.5 \%$ in adolescents. The rate of

pre-diabetes is as high as $40-50 \%$ in the population under 40 years old(Xu et al. 2013). It has

been reported that the pathogenesis of youth T2DM is mainly related to the rapid decline of islet

$\beta$ - cell function and obesity-induced IR. In young individuals with obesity and T2DM, the levels

of circulating inflammatory factors, adipokines, hepatocytes, and myokines were significantly

changed(Lascar et al. 2018; Reinehr et al. 2016), but the underlying mechanisms are unclear.

Serum Fetuin-B is considered to be an adipo-hepatokine and a member of the cystatin

superfamily of cysteine protease inhibitors. It is mainly secreted by the liver, and a small amount is also secreted by white adipose tissue (WAT) and myocardial cells(Olivier et al. 2000). The

results from Meex et al. showed that Fetuin-B could induce IGT by inhibiting the effect of

insulin on myotubes and hepatocytes in mice. In obese mice, knockout of the Fetuin-B gene

significantly improved impaired glucose tolerance (IGT). In an in vivo study, it was shown that

Fetuin-B may regulate blood glucose through a non-insulin signaling pathway, but the specific

mechanism is still unclear (Meex et al. 2015). In a clinical study, Qu et al. found that the serum

Fetuin-B levels in T2DM patients were significantly higher than those in IGT and normal

subjects, and serum Fetuin-B was correlated with TG, fasting insulin, homeostasis model 
85

86

87

88

89

90

91

2018). In addition, it has been reported that serum Fetuin-B levels are significantly increased in patients with nonalcoholic fatty liver disease (NAFLD), and related to IR (Li et al. 2018). In contrast, a recent study showed that the mRNA expression of Fetuin-B in the liver was not associated with HOMA-IR(Peter et al. 2018). Therefore, the relationship between serum FetuinB and IR is still controversial.

In this study, the changes in serum Fetuin-B were observed in simple IR, IGT, and normal subjects, and the relationship between serum Fetuin-B and other metabolic indexes was evaluated. In addition, because off-label use of liraglutide (Lira) has been proposed for the management of PCOS in women with overweight/obesity-related IR, therefore, in a subgroup of overweight/obese women, the effects of Lira on Fetuin-B and glucose metabolism were investigated.

\section{Research population and methods}

\section{Research population}

A total of 304 young women were enrolled in this study (Fig. 1), including 80 newly diagnosed

IR patients (IR-NG group), 97 individuals with impaired glucose tolerance (IGT group), and 127 healthy women (NGT group). The individuals were recruited from the outpatient department of Endocrinology or from daily physical examination, the Second Affiliated Hospital of Chongqing 
106

107

108

109

110

111

112

113

114

115

116

117

118

119

120

121

122

123

124

125

126

Medical University from January 2017 to December 2019. IGT was diagnosed according to the

American Diabetes Association (ADA)(American Diabetes 2012). The presence of pre-diabetes

was defined by the occurrence of IGT at 2-hours oral glucose tolerance (2h-OGTT). M-value $<$

6.28 was considered to have IR(Wei-Gang et al. 2009). Individuals with M-value $<6.28$ and

normal fasting blood glucose (FBG) levels were included in the IR-NG group. All IGT and IR

patients were newly diagnosed without any medication or lifestyle intervention. The exclusion

criteria included type 1 diabetes mellitus (T1DM), T2DM, hypertension, and other important

organ diseases such as heart, liver, and kidney. NGT group had normal blood glucose [FBG $<$

$5.1 \mathrm{mmol}$; 2-h blood glucose post-glucose load $(2-\mathrm{h} \mathrm{BG})<7.8 \mathrm{mmol}$, no family history of

diabetes and hypertension, no chronic diseases, and medication recently. All subjects signed

informed consent before the experiment. This study was conducted according to the declaration

of Helsinki and approved by the ethics committee of the Second Affiliated Hospital of

Chongqing Medical University (2014 Colombo Review No. (72)). Human research was

registered at the Chinese Clinical Trial Registry (registered 23 June 2011), chictr.org.cn

(CHICTR-OCC-11001422).

\section{Anthropometric and biochemical measurements}

After fasting for 10-12 h, all subjects were given a physical examination and biochemical tests

by professionals. The physical examination included body weight, blood pressure (BP), waist

circumference (WC), and body fat content (Fat \%). Biochemical parameters included total

cholesterol (TC), triglyceride (TG), high-density lipoprotein cholesterol (HDL-C), low-density

lipoprotein cholesterol (LDL-C), free fatty acid (FFA), HbA1c, FBG, 2-hour postprandial blood

PeerJ reviewing PDF | (2020:12:56524:2:1:NEW 30 Jun 2021) 
127 glucose (2h-BG), fasting insulin (FIns), and 2-hour postprandial insulin (2h- INS), as previously

$128 \operatorname{reported}($ Xu et al. 2020).

129 Determination of serum Fetuin-B concentration

130 Serum Fetuin-B concentration was determined by an ELISA kit (RayBiotech, Inc. Norcross, GA,

131 USA). The detection limit for Fetuin-B was $4.0 \mathrm{ng} / \mathrm{ml}$. Intra- and inter-assay variations (CV)

132 were $10 \%$ and $12 \%$, respectively. This method has high sensitivity, good specificity, and no

133 obvious cross-reaction.

134 OGTT and euglycemic-hyperinsulinemic clamp test (EHC)

135 After 10-12 hours of fasting overnight, all subjects were given $75 \mathrm{~g}$ glucose orally and the OGTT 136 test was performed at 8:00 AM(American Diabetes 2012). During the OGTT, blood samples 137 were collected for the measurements of blood glucose, insulin, and serum Fetuin-B at 0, 30, 60, and $120 \mathrm{~min}$. The EHC was performed in all subjects. The operation process was as previously reported $(X u$ et al. 2020). During the EHC, human insulin (1mU/kg/min) was continuously infused for 2 hours. At the same time, the infusion rate of the $20 \%$ glucose was adjusted according to the blood glucose levels, tested every $15 \mathrm{~min}$, to maintain blood glucose at the basic level (4.5-5.5 mmol/L). In the steady-state of the clamp, GIR was equal to glucose disposal rate (GDR), and M-value was related to GIR and body weight. Blood samples were collected at 0, 80, 100, and 120 min for the determination of Fetuin-B and insulin. Blood samples were centrifuged, and serum was separated and stored at $-80^{\circ} \mathrm{C}$ for subsequent analysis.

GLP-1RA intervention

147 A total of 26 overweight/obese women with IR participated in GLP-1RA intervention for 24 
148 149 150

weeks. These women were enrolled for intervention with lira due to ovarian dysfunction and refractory menstrual problems. Liar, a GLP-1RA, was injected subcutaneously once a day. The dose increased from $0.6 \mathrm{mg}$ to $1.8 \mathrm{mg}$. Inclusion criteria included age of 18-35 and BMI of 25-35

$\mathrm{kg} / \mathrm{m}^{2}$. Exclusion criteria included the family history of thyroid tumor, the history of medullary thyroid carcinoma, severe gastrointestinal disease, acute pancreatitis, and pregnancy. Those subjects did not use any drugs in the last 3 months. Physical examination and biochemical tests, OGTT, and EHC tests were performed before treatment, 12 and 24 weeks after treatment. All subjects signed informed consent before treatment.

\section{Calculation formula}

$\mathrm{BMI}=$ body weight/height $2, \mathrm{WHR}=\mathrm{WC} /$ hip circumference $(\mathrm{HC})$, HOMA2-IR was calculated by the software (HOMA calculator v2.2.2)(Wallace, et al 2004), M-value = GIR/body weight, and M-value $<6.28$ is considered to have IR(Wei-Gang, et al. 2009).

\section{Statistical analysis}

SPSS version 24.0 software (SPSS 2.0, Chicago, IL) was used for statistical analysis. Data were expressed as mean $\pm \mathrm{SD}$ or median. Variables that are not normally distributed were converted logarithmically. Analysis of variance was used for comparison among groups, and Fisher's Least Significant Difference (LSD) test was used for comparison between two groups. The relationship between serum Fetuin-B and other variables was evaluated by linear regression analysis with controlled covariates. Multiple linear regression analysis was used to identify independent factors associated with serum Fetuin-B. Multiple logistic regression analysis was conducted to understand the association of serum Fetuin-B with IR and IGT. ROC curves were constructed to 
169

170

171

172

173

174

175

176

177

178

179

180

181

182

183

184

185

186

187

188

189

174

evaluate the sensitivity and specificity of serum Fetuin-B in predicting IR and IGT.

We calculated tolerance and variance inflation factor (VIF) values to evaluate multicollinearity

between variables. A tolerance $<0.1$ and VIF $>10$ is considered indicative of multicollinearity.

Compared with the control group, $p<0.05$ was considered to be statistically significant.

3

175

176

77

78

79

\section{Results}

\section{Serum Fetuin-B levels and biochemical parameters in the study population}

Table 1 shows the clinical biochemical indexes and circulating Fetuin-B levels in each group.

We found that BMI, Fat \%, WC, BP, TG, TC, LDL, HbA1c, FBG, 2 h-BG, FIns, 2 h-INS,

HOMA2-IR, the area under the curve of glucose (AUCg) and insulin (AUCi) in IR-NG and IGT groups were significantly higher than those in the control group (all $\mathrm{p}<0.01$ ), while HDL-C and M-values were lower $(\mathrm{p}<0.01)$. In addition, FFA, 2h-BG, 2h-Ins, and AUCg in the IGT group were significantly higher than those in the IR-NG group $(p<0.05$ or $p<0.01)$. Serum Fetuin-B levels in IGT and IR-NG groups were significantly higher than those in normal controls $(\mathrm{p}<$ 0.05 or $\mathrm{p}<0.01$, Table 1 and Fig. $2 \mathrm{~A}$ ). Fetuin-B levels were higher in the IGT group than those 
190

191

192

193

194

195

196

197

198

199

200

201

202

203

204

205

206

207

208

209

in the IR-NG group (Fig. 2A, $p<0.05$ ). Furthermore, serum Fetuin-B levels in obese/overweight women $\left(\mathrm{BMI} \geq 24 \mathrm{~kg} / \mathrm{m}^{2}\right)$ were significantly higher than those in normal controls $(7.63 \pm 0.38 v s$.

$5.95 \pm 0.32 \mathrm{mg} / \mathrm{L}, p<0.01$ ) (Fig. 2B). The relationship between serum Fetuin-B and IR was analyzed by combing IR-NG and IGT individuals into the IR group. The results showed that serum Fetuin-B levels were significantly correlated with IR (Table S2).

\section{Association of serum Fetuin-B with clinical and biochemical indexes in young women}

In all study subjects, serum Fetuin-B levels were positively correlated with obesity and IRrelated parameters (BMI, fat\%, WC, TG, FIns, 2h-INS, AUCi, AUCg, HOMA2-IR, and Mvalue) (all $\mathrm{p}<0.01$ ), but not with TC, HDL-C, LDL-C and FFA (Table 2). After controlling age and Fat $\%$, serum Betuin-B levels were still significantly positively correlated with WC, FIns, HOMA2-IR, and M-value ( $\mathrm{p}<0.05$ or $\mathrm{p}<0.01$, Table 2). Multiple regression analysis showed that M-value was an independent factor affecting serum Fetuin-B (Fig. 2C, Table 2). The regression equation is $\mathrm{Y}_{\text {Fetuin-B }}=9.231-0.379 \mathrm{X} \mathrm{M}$-value.

\section{Association of serum fetuin-B with IR and IGT in women}

By multiple logistic regression analysis, we found that serum Fetuin-B levels were significantly correlated with the occurrence of IR-NG and IGT (IR-NG: OR, 1.13; 95\% CI, 1.04-1.22; $p<$ 0.01; IGT: OR, 1.20; 95\% CI, 1.09-1.27, $p<0.01)$. After control of age, Fat \%, BMI, WC, BP, and TG, serum Fetuin-B levels were still correlated with IGT ( $p<0.05$ or $p<0.01$, Table S1), while by controlling age only, Fetuin-B was correlated with the occurrence of IR in women ( $p<$ 0.01 , Table S2). 

increased with the increase of serum Fetuin-B concentration.

\section{ROC curve analysis}

217 To evaluate the predictive value of serum fetuin-B levels for IGT and IR, ROC curves were

value of Fetuin-B for predicting IR was $5.44 \mathrm{mg} / \mathrm{L}$ (Fig. S1B).

\section{Effect of the OGTT and EHC on serum Fetuin-B levels in women}

To further explore the regulatory factors of serum fetuin-B level, we performed the OGTT and

EHC experiments. During the OGTT, serum Fetuin-B levels in the NGT group were significantly increased (Fig. 3A) and reached the peak at 30 minutes (11.42 $\pm 1.0 \mathrm{mg} / \mathrm{L}$ vs. 3.42

$226 \pm 0.57 \mathrm{mg} / \mathrm{L}, \mathrm{p}<0.01)$, but no change in both IR-NG and IGT groups. The areas under the 
231 levels increased gradually following the stimulation of hyperinsulinemia (from $6.29 \pm 0.99 \mathrm{mg} / \mathrm{L}$

232 to $10.18 \pm 1.30 \mathrm{mg} / \mathrm{L}$ ) (Fig. 3D). Interestingly, there was no significant change in serum Fetuin-B

233 levels in both IR-NG and NGT groups during the EHC, suggesting that serum Fetuin-B levels

234 were regulated by insulin in different glucose tolerance populations.

235

236

237

238

239

240

241

242

243

244

245

246

247

248

249

250

251

\section{7}

\section{8}

\section{Effect of GLP-1RA treatment on serum Fetuin-B level in obese individuals}

26 overweight/obese women with IR (BMI, $28.4 \pm 0.6 \mathrm{~kg} / \mathrm{m} 2)$ were treated with a Liar for 24

weeks. The changes in general clinical and biochemical indexes before and after treatment are

shown in Table 3. After the treatment, obesity, and lipid metabolism-related indicators (BMI, Fat

\%, WC and TG), glucose metabolism and IR-related indicators (FBG, FIns, HbA1c, HOMA2-

IR) were significantly improved compared with those before treatment $(p<0.01$ or $p<0.05$;

Table 3). In addition, Liar treatment significantly decreased the M-values of the EHC in these obese women, which further indicates the improvement of IR (Fig. 4A). Importantly, serum

Fetuin-B levels were significantly reduced after the 24 weeks of GLP-1RA intervention in these obese women with IR (from $8.08 \pm 1.77 \mathrm{mg} / 1$ to $6.75 \pm 1.57 \mathrm{mg} / \mathrm{l}, \mathrm{p}<0.05$; Fig. 4B).

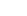




\section{Discussion}

In this study, the changes in serum Fetuin-B concentrations in women with IGT or IR alone were reported for the first time. We found that 1) serum Fetuin-B levels in women with IGT or IR alone were significantly higher than those in the normal control group and significantly correlated with glucose and lipid metabolism; 2) in these individuals, the M-value was an independent influencing factor for serum Fetuin-B levels; 3) with the increase of serum Fetuin-B levels, the risk of IR and IGT was also increased; 4) The response of serum Fetuin-B to hyperglycemia and hyperinsulinemia was different in different glucose tolerance population; 5) GLP-1RA treatment not only improved IR but also reduced serum Fetuin-B levels. These results suggest that Fetuin-B may be a biomarker related to IR and glucose and lipid metabolism. In recent years, there have been some reports that Fetuin-B is related to metabolic diseases. 
273 lipids (Meex et al. 2015). Other studies also found that serum Fetuin-B levels were significantly

274 increased in T2DM, NAFLD, and GDM patients, and were associated with IR and obesity

275 (Kralisch et al. 2017; Li et al. 2018). In contrast, some studies found that serum Fetuin-B levels

276 were not related to BMI (Qu et al. 2018), and there was no significant correlation between serum

277 Fetuin-B and hepatocyte fat content in NAFLD patients (Ebert et al. 2017). Therefore, the results

278 from previous studies have not been consistent. However, most of the previous studies were

279 cross-sectional and descriptive studies. They were merely a preliminary exploration of Fetuin-B, and there was no intervention experiment to fully prove the relationship between Fetuin-B and human IR, and no state-of-the-art methodology was used. In addition, there have been many confounding factors in previous studies, such as middle-aged and elderly subjects, most of whom had long-term dyslipidemia or other metabolic diseases, and with a long history of drug treatment. In addition, age and gender also have important effects on obesity and IR. Therefore, if applied to clinical work, the previous results have some limitations.

In the present study, we further confirm that serum Fetuin-B levels were significantly increased in women with IR and IGT, and significantly correlated with BMI, HOMA-IR, and glucose and lipid metabolism. This was consistent with the results of Adamska et al. in the PCOS population (Adamska et al. 2019). Based on the following reasons, we believe that our results are accurate and reliable: 1) we selected young subjects as the research objects and thus avoided age-related IR; 2) we chose women as the research subjects and avoided the influence of sex hormones on insulin sensitivity in vivo; 3) our subjects did not use drug treatments or lifestyle interventions, avoiding interventional influence on serum Fetuin-B levels. 
In the current study, there is a contradictory finding that the cut-off value of Fetuin-B for

295

296

297

predicting IR was higher than that of predicting IGT. The reason for this phenomenon remains unknown. We speculate that serum Fetuin-B levels may be mainly affected by insulin, and there was no hyperinsulinemia in a small number of the IGT individuals. In addition, IGT individuals with IR were also included in the calculation of the IR cut-off value.

To further explore the regulation of glucose and insulin on Fetuin-B levels, we conducted an oral OGTT and an EHC. Under OGTT induced hyperglycemia and hyperinsulinemia, the serum Fetuin-B levels in the control group increased significantly at different times, while the serum Fetuin-B levels in IGT and IR-NG groups did not change significantly. We speculate that in individuals with IGT or IR, long-term IR results in the decline or failure of Fetuin-B secretion function in tissues and cells. Therefore, there was no response to high glucose and high insulin stimulation. However, it is not known if hyperglycemia or/and hyperinsulinemia stimulates the secretion or release of Fetuin-B in vivo. Therefore, we performed the EHC study, a gold standard for IR evaluation, and found that acute hyperinsulinemia resulted in significantly elevated circulating Fetuin-B levels in IGT subjects under normal glucose conditions. However, acute hyperinsulinemia did not cause changes in circulating Fetuin-B in IR-NG or control groups. We, therefore, believe that in the normal population, acute hyperglycemia increases the release and secretion of Fetuin-B in vivo, while hyperinsulinemia does not stimulate the secretion of FetuinB. However, the underlying mechanism needs further study. Metformin has been widely reported for the treatment of obesity and polycystic ovary syndrome (PCOS) (Gilbert et al.2006; Cassina et al. 2014). Although metformin can effectively 
315 reduce blood glucose, its effect of reducing body weight and improving IR is relatively limited

316 (Li et al, 2015). Recently, GLP-1RA has been used for the treatment of obese individuals, and it

317 has been observed that GLP-1RA is superior to metformin in improving IR, but there is still

318 controversy on its effect on body weight reduction (Mirabelli et al. 2019; Lin et al. 2020). In

319 addition, GLP-1 has been found to stimulate or inhibit adipokine releases, such as adiponectin

and visfatin (Li et al. 2011; Li et al. 2008). To further observe the effect of GLP-1RA and insulin

sensitivity on circulating Fetuin-B, we treated overweight/obese patients with Lira, a GLP-1RA,

for 24 weeks, and observed the changes in serum Fetuin-B levels. After 24 weeks of Lira

treatment, circulating Fetuin-B levels in IGT individuals decreased significantly with weight loss

and increased with insulin sensitivity. It has been well known that Lira is generally used at doses

up to $1.8 \mathrm{mg}$ as an anti-diabetic agent, but higher doses (up to $3.0 \mathrm{mg}$ ) have been approved for

weight loss in the US and Europe in obese individuals, irrespective of hyperglycemia. Given the

weight characteristics of the Chinese population, we treated IR patients with $1.8 \mathrm{mg}$ Lira for 24

weeks. The results showed that body weight was also significantly reduced at this dose.

Interestingly, menstrual cycles was not significantly changed in our study, maybe because of the

small number of participants. And we are recruiting a larger scale of patients in another trial to

further study that. Based on these results, we speculate that the effect of Lira on Fetuin-B is

likely a result of the weight loss and/or metabolic changes and improved IR after Lira treatment.

so we can not infer the causal relationship between Fetuin-B and the occurrence and 
336

337

338

339

340

341

342

343

344

345

346

347

348

349

350

351

352

353

354

355

356

not be applicable to men and other ethnic populations. 3) A small sample size, especially in an intervention trial, may lead to biased results; 4) as a secretory protein, serum Fetuin-B may be secreted by pulse, and the results of a blood sample may not fully reflect the true levels of circulating Fetuin-B in vivo; 5) Lira treatment was a self-control experiment with no placebo control group. Nevertheless, we believe that our results are still sufficient to prove the association between Fetuin-B and IR and guide other studies.

\section{Conclusions}

Our results suggest that serum Fetuin-B levels were elevated in young women with IGT or IR and might be associated with glucose metabolism and IR. In addition, the secretion and release of Fetuin-B may be regulated by blood glucose. Therefore serum Fetuin-B may be a biomarker for pre-diabetes and IR in young women.

\section{Acknowledgments}

We thank patients and healthy individuals who made this study possible.

Financial Support: This work was supported by research grants from the National Natural Science Foundation of China (No. 81670755), and the Natural Science Foundation of Chongqing (No. cstc2017jcyjAX0050).

Author Contributions: X.X. and S.X. researched and analyzed the data. G.Y. performed the statistical analysis. H.L. and C.C. reviewed and edited the manuscript. L.L. was the guarantors of this work and as such had full access to all the data in the study and take responsibility for the integrity of the data and the accuracy of the data analysis.

Disclosure Summary: The authors have nothing to disclose. The authors declare that they have 
357

358

359

360

361

362

363

364

365

366

367

368

369

370

371

372

373

374

375

376

377

378

379

380

381

382

383

384

385

386

387

388

389

390

391

392

393

no conflict of interest.

\section{Reference}

Arcidiacono B, Chiefari E, Foryst-Ludwig A, Currò G, Navarra G, Brunetti FS, Mirabelli M, Corigliano DM, Kintscher U, Britti D, Mollace V, Foti DP, Goldfine ID, Brunetti A. 2020. Obesity-related hypoxia via miR-128 decreases insulin-receptor expression in human and mouse adipose tissue promoting systemic insulin resistance. EBioMedicine. 59:102912 DOI: 10.1016/j.ebiom.2020.102912.

Adamska A, Polak A, Krentowska A, Łebkowska A, Hryniewicka J, Leśniewska M, Kowalska I. 2019. Increased serum fetuin-B concentration is associated with HOMA- $\beta$ and indices of liver steatosis in women with polycystic ovary syndrome: a pilot study. Endocrine Connections 8:1159-1167 DOI: 10.1530/ec-19-0243

American Diabetes A. 2012. Diagnosis and classification of diabetes mellitus. Diabetes Care 35 Suppl 1:S64-71 DOI: 10.2337/dc12-s064

Chatterjee S, Khunti K, Davies MJ. 2017. Type 2 diabetes. Lancet 389:2239-2251 DOI: $10.1016 / \mathrm{s} 0140-6736(17) 30058-2$

Cassina M, Donà M, Di Gianantonio E, Litta P, Clementi M. 2014. First-trimester exposure to metformin and risk of birth defects: a systematic review and meta-analysis. Hum Reprod Update. 20:656 - 669 DOI: 10.1093/humupd/dmu022

Ebert T, Linder N, Schaudinn A, Busse H, Berger J, Lichtinghagen R, Keim V, Wiegand J, Karlas T. 2017. Association of fetuin B with markers of liver fibrosis in nonalcoholic fatty liver disease. Endocrine 58:246-252 DOI: 10.1007/s12020-017-1417-z

Gilbert C, Valois M, Koren G. 2006. Pregnancy outcome after first-trimester exposure to metformin: a meta-analysis. Fertil Steril 86:658-663 doi: 10.1016/j.fertnstert.2006.02.098.

Kralisch S, Hoffmann A, Lössner U, Kratzsch J, Blüher M, Stumvoll M, Fasshauer M, Ebert T. 2017. Regulation of the novel adipokines/ hepatokines fetuin A and fetuin B in gestational diabetes mellitus. Metabolism: clinical and experimental 68:88-94 DOI: 10.1016/j.metabol.2016.11.017 
394

395

396

397

398

399

400

401

402

403

404

405

406

407

408

409

410

411

412

413

414

415

416

417

418

419

420

421

422

423

424

425

426

427

428

429

430

431

432

433

434
Lascar N, Brown J, Pattison H, Barnett AH, Bailey CJ, Bellary S. 2018. Type 2 diabetes in adolescents and young adults. The Lancet Diabetes \& Endocrinology 6:69-80 DOI: 10.1016/ s2213- 8587(17)30186-9

Li L, Miao Z, Liu R, Yang M, Liu H, Yang G. 2011. Liraglutide prevents hypoadiponectinemiainduced insulin resistance and alterations of gene expression involved in glucose and lipid metabolism. Molecular medicine (Cambridge, Mass) 17:1168-1178 DOI: 10.2119/molmed.2011.00051

Li L, Yang G, Li Q, Tan X, Liu H, Tang Y, Boden G. 2008. Exenatide prevents fat-induced insulin resistance and raises adiponectin expression and plasma levels. Diabetes, obesity \& metabolism 10:921-930 DOI: 10.1111/j.1463-1326.2007.00832.x

Li M, Yang M, Zhou X, Fang X, Hu W, Zhu W, Wang C, Liu D, Li S, Liu H, Yang G, Li L. 2015. Elevated circulating levels of irisin and the effect of metformin treatment in women with polycystic ovary syndrome. J Clin Endocrinol Metab.100:1485-1493. doi: $10.1210 /$ jc. 2014-2544.

Li Z, Lin M, Liu C, Wang D, Shi X, Chen Z, Liu Y, Yang S, Li X. 2018. Fetuin-B links nonalcoholic fatty liver disease to type 2 diabetes via inducing insulin resistance: Association and path analyses. Cytokine 108:145-150 DOI: 10.1016/j.cyto.2018.03.023

Meex RC, Hoy AJ, Morris A, Brown RD, Lo JC, Burke M, Goode RJ, Kingwell BA, Kraakman MJ, Febbraio MA, Greve JW, Rensen SS, Molloy MP, Lancaster GI, Bruce CR, Watt MJ. 2015. Fetuin B Is a Secreted Hepatocyte Factor Linking Steatosis to Impaired Glucose Metabolism. Cell Metab 22:1078-1089 DOI: 10.1016/j.cmet.2015.09.023

Mirabelli M, Chiefari E, Caroleo P, Arcidiacono B, Corigliano DM, Giuliano S, Brunetti FS, Tanyolaç S, Foti DP, Puccio L, Brunetti A. 2019. Long-Term Effectiveness of Liraglutide for Weight Management and Glycemic Control in Type 2 Diabetes.Int J Environ Res Public Health. 17: 207 DOI: 10.3390/ijerph17010207

Olivier E, Soury E, Ruminy P, Husson A, Parmentier FO, Daveau M, Salier JP. 2000. Fetuin-B, a second member of the fetuin family in mammals. Biochemical Journal 350 Pt 2:589597

Peter A, Kovarova M, Staiger H, Machann J, Schick F, Königsrainer A, Königsrainer I, Schleicher E, Fritsche A, Häring H-U, Stefan N. 2018. The hepatokines fetuin-A and fetuin-B are upregulated in the state of hepatic steatosis and may differently impact on glucose homeostasis in humans. American Journal of Physiology-Endocrinology and Metabolism 314:E266-E273 DOI: 10.1152/ajpendo.00262.2017

Qu H, Qiu Y, Wang Y, Liao Y, Zheng Y, Zheng H. 2018. Plasma fetuin-B concentrations are associated with insulin resistance and first-phase glucose-stimulated insulin secretion in 
435

436

437

438

439

440

441

442

443

444

445

446

447

448

449

450

451

452

453

454

455

456

457

458

459

460

461

462

463

464

465

466

467

468

469

individuals with different degrees of glucose tolerance. Diabetes Metab 44:488-492 DOI: 10.1016/j.diabet.2018.02.003

Reinehr T, Karges B, Meissner T, Wiegand S, Stoffel-Wagner B, Holl RW, Woelfle J. 2016. Inflammatory Markers in Obese Adolescents with Type 2 Diabetes and Their Relationship to Hepatokines and Adipokines. $J$ Pediatr 173:131-135 DOI: 10.1016/j.jpeds.2016.02.055

Wallace T, Levy J, Matthews D. 2004. Use and abuse of HOMA modeling. Diabetes Care 27:1487-1495 DOI: 10.2337/diacare.27.6.1487

Wei-Gang Z, Tao Y, Qi S, Yong FU, Guo-Hua Y, Ying-Yue D, Ya-Xiu D. 2009. Establishment of hyperinsulinemic euglycemic clamp technique and the reference value of insulin sensitivity index in Chinese. Chinese Journal of Clinical Nutrition

Xu X, Zhang T, Mokou M, Li L, Li P, Song J, Liu H, Zhu Z, Liu D, Yang M, Yang G. 2020. Follistatin-like 1 as a Novel Adipomyokine Related to Insulin Resistance and Physical Activity. The Journal of clinical endocrinology and metabolism 105 DOI: 10.1210/clinem/dgaa629

Xu Y, Wang L, He J, Bi Y, Li M, Wang T, Wang L, Jiang Y, Dai M, Lu J, Xu M, Li Y, Hu N, Li J, Mi S, Chen CS, Li G, Mu Y, Zhao J, Kong L, Chen J, Lai S, Wang W, Zhao W, Ning G, China Noncommunicable Disease Surveillance G. 2013. Prevalence and control of diabetes in Chinese adults. JAMA 310:948-959 DOI: 10.1001/jama.2013.168118

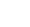

(1)

2

63


Figure 1

\section{Study flow diagram}

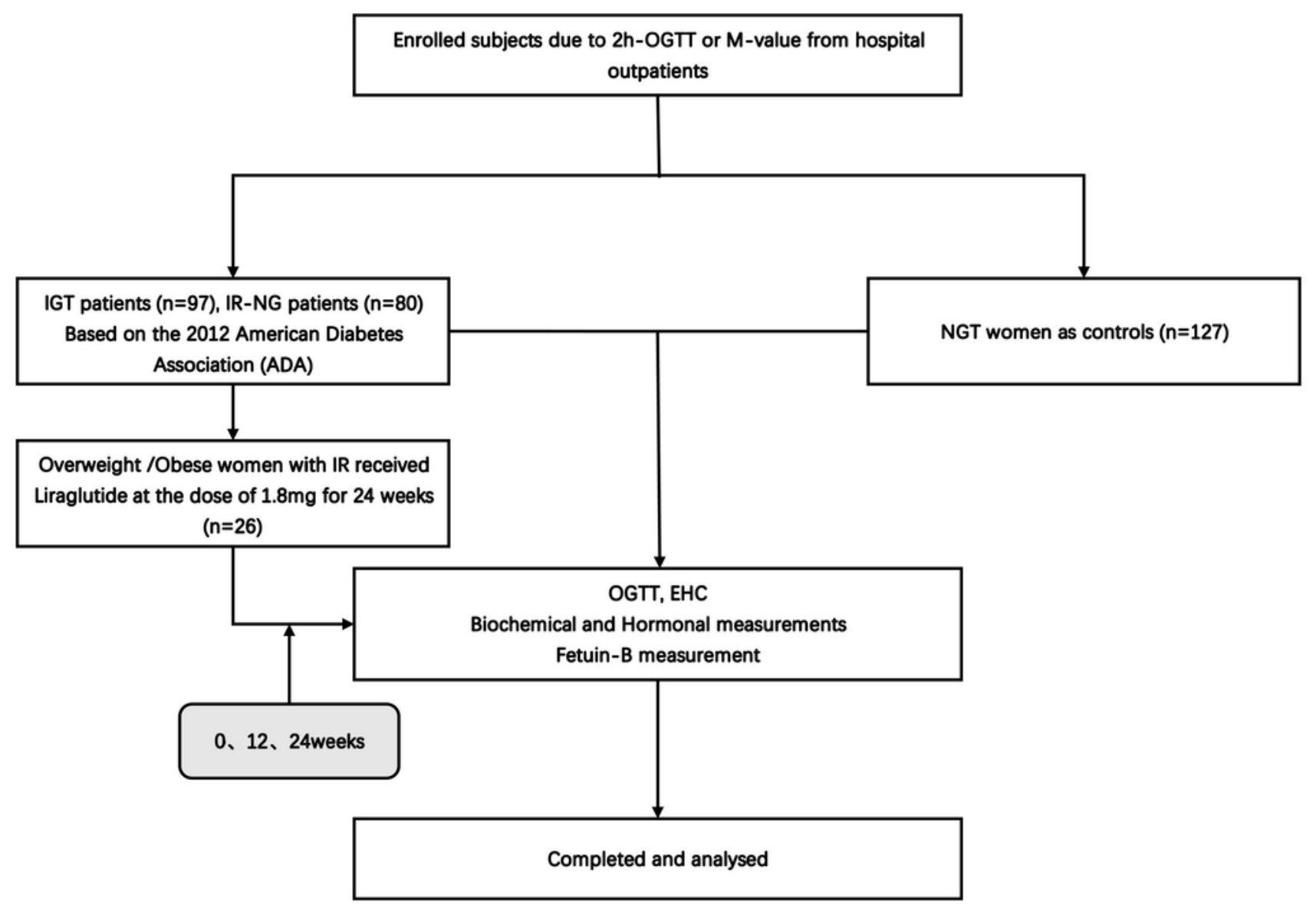




\section{Figure 2}

Serum fetuin-B levels in the study population.

(A) Circulating fetuin-B levels in NGT, IR-NG and IGT subjects. (B) Circulating fetuin-B levels according to BMI (lean: BMI $<24 \mathrm{~kg} / \mathrm{m} 2$; overweight/obese: $\mathrm{BMI} \geq 24 \mathrm{~kg} / \mathrm{m} 2$ ). (C) Circulating fetuin-B levels, according to M-values (IR: M-values < 6.28; non-IR: M-values $\geq 6.28$ ). (D) All factors and stepwise multiple regression analyses of the serum fetuin-B in study individuals. (E) The odds ratio of having IR-NG in different quartiles of serum fetuin-B ( quartile 1 :

0.85-2.12 mg/L; quartile 2: 2.13-6.35 mg/L; quartile 3: 6.36-8.61 mg/L; quartile 4: >8.61 $\mathrm{mg} / \mathrm{L}$ ). (F) The odds ratio of having IGT in different quartiles of serum fetuin-B (quartile 1 : 0.85-3.39 mg/L; quartile 2: 3.40-6.69 mg/L; quartile 3: $6.70-8.99 \mathrm{mg} / \mathrm{L}$; quartile 4: $>8.99 \mathrm{mg} / \mathrm{L}$ ) Data were means \pm SME. $* p<0.05$ or $* * p<0.01$ vs. Controls, lean, no-IR or quartile 1 . 
A

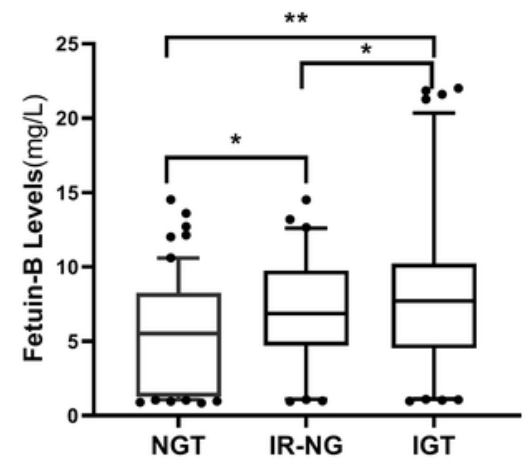

B

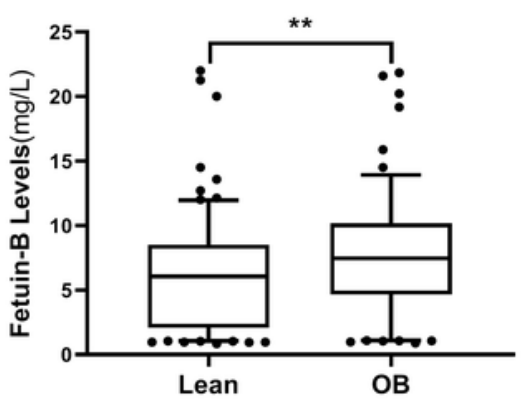

E
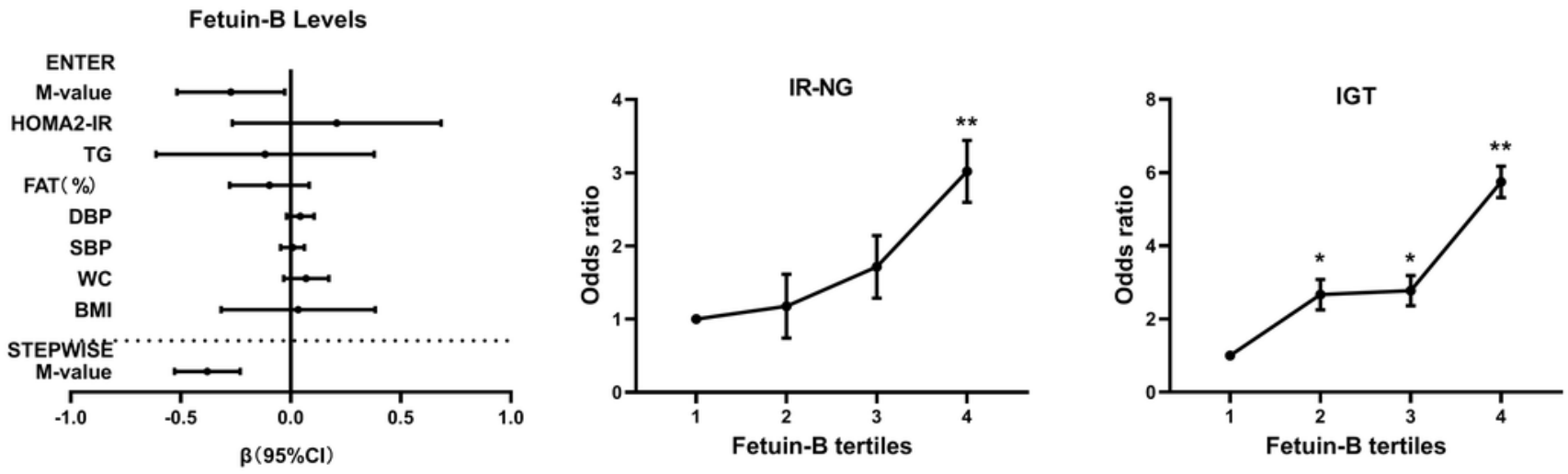
Figure 3

Circulating fetuin-B levels in interventional studies.

(A) Time course of changes in circulating fetuin-B levels in healthy, IR-NG and IGT subjects during the OGTT; $(B)$ The area under the curve for serum Fetuin- $B\left(A \cup C_{f}\right)$ during the OGTT. (C) The M-values in healthy, IR-NG and IGT subjects during the EHC. (D)Time course of circulating fetuin-B changes in healthy, IR-NG and IGT subjects during the EHC; Data were meant \pm SME. $* p<0.05$ or $* * p<0.01$ vs. Control or baseline.

A

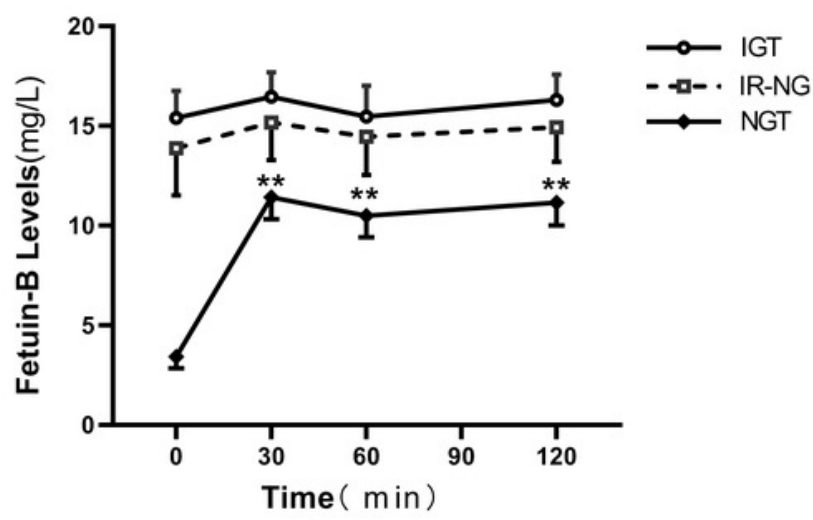

C

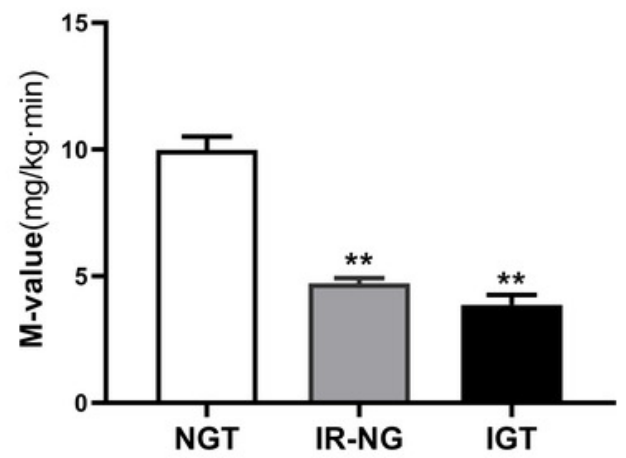

B

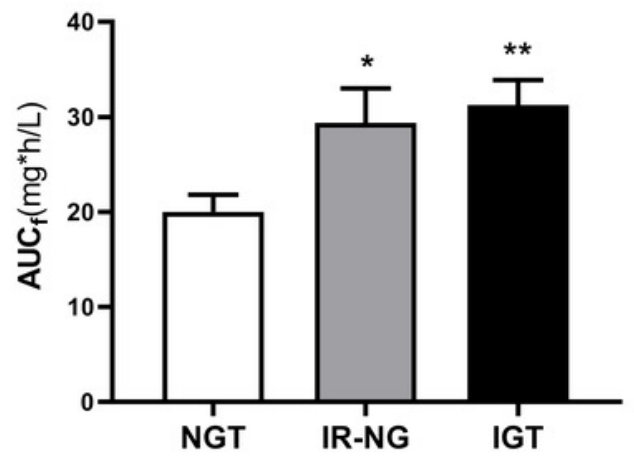

D

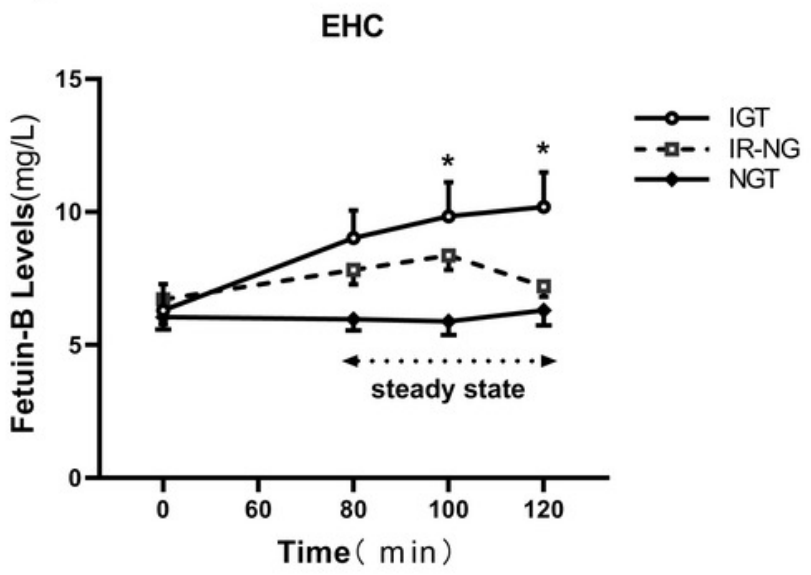


Figure 4

Effects of GLP-1RA treatment on serum fetuin-B in young women.

(A) Changes of M-value in IGT or IR subjects during the EHC after GLP-1RA treatment. (B) Serum fetuin-B levels in IGT or IR subjects after GLP-1RA treatment. Data were means \pm SME. $* p<0.05$ or $* * p<0.01$ vs. Baseline.

A

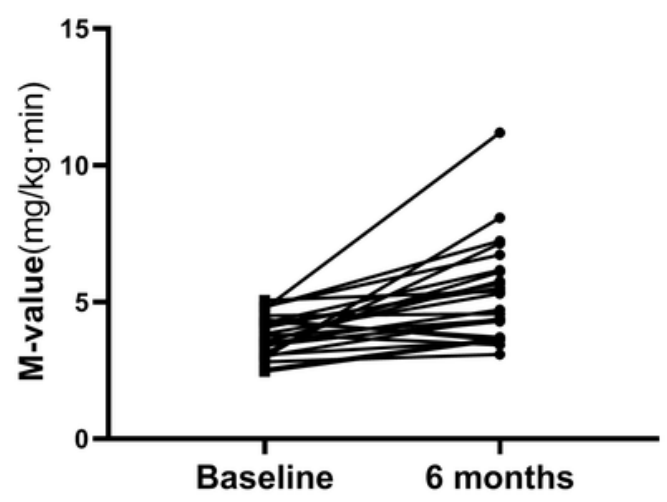

B

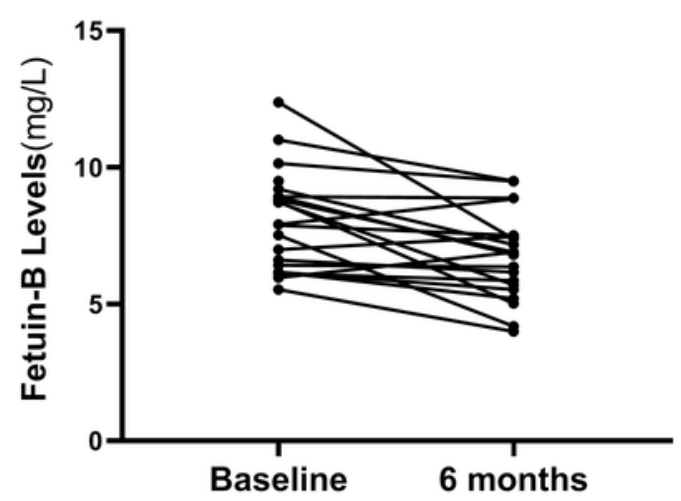




\section{Table 1 (on next page)}

Anthropometrics and metabolic parameters in study population 


\begin{tabular}{|c|c|c|c|}
\hline Variable & NGT $(n=127)$ & IR-NG $(n=80)$ & IGT $(n=97)$ \\
\hline Age (years) & $26.5 \pm 3.0$ & $27.2 \pm 4.5$ & $27.5 \pm 4.0$ \\
\hline BMI $\left(\mathrm{kg} / \mathrm{m}^{2}\right)$ & $20.2 \pm 2.2$ & $26.3 \pm 3.6^{* *}$ & $26.1 \pm 4.2^{* *}$ \\
\hline FAT $(\%)$ & $26.5 \pm 5.1$ & $38.0 \pm 5.9^{* *}$ & $37.4 \pm 6.6^{* *}$ \\
\hline $\mathrm{WC}(\mathrm{cm})$ & $69.5 \pm 7.0$ & $86.1 \pm 8.9^{* *}$ & $86.5 \pm 9.7^{* *}$ \\
\hline $\mathrm{SBP}(\mathrm{mmHg})$ & $107.9 \pm 8.5$ & $116.5 \pm 11.4^{* *}$ & $116.4 \pm 12.9^{* *}$ \\
\hline $\mathrm{DBP}(\mathrm{mmHg})$ & $71.4 \pm 9.1$ & $74.7 \pm 9.4^{*}$ & $75.0 \pm 9.5^{* *}$ \\
\hline $\mathrm{TC}(\mathrm{mmol} / \mathrm{L})$ & $3.88 \pm 0.90$ & $4.40 \pm 0.79^{* *}$ & $4.60 \pm 1.02^{* *}$ \\
\hline $\mathrm{TG}(\mathrm{mmol} / \mathrm{L})^{\dagger}$ & $0.81(0.60-1.13)$ & $1.48(1.04-2.12)^{* *}$ & $1.59(1.21-2.14)^{* *}$ \\
\hline HDL-C $(\mathrm{mmol} / \mathrm{L})^{\dagger}$ & $1.24(0.99-1.51)$ & $1.10(0.94-1.28)$ & $1.17(1.02-1.30)^{* *}$ \\
\hline LDL-C (mmol/L) & $2.20 \pm 0.76$ & $2.60 \pm 0.56^{* *}$ & $2.78 \pm 0.86^{* *}$ \\
\hline FFA $(\mu \mathrm{mol} / \mathrm{L})^{\dagger}$ & $0.51(0.36-0.73)$ & $0.48(0.37-0.65)$ & $0.56(0.45-0.78)^{* * \Delta}$ \\
\hline $\operatorname{HbA1c}(\%)^{\dagger}$ & $5.10(5.00-5.30)$ & $5.40(5.20-5.60)^{* *}$ & $5.50(5.30-5.80)^{* *}$ \\
\hline $\mathrm{FBG}(\mathrm{mmol} / \mathrm{L})^{\dagger}$ & $4.54(4.26-4.91)$ & $5.26(4.98-5.50)^{* *}$ & $5.35(5.04-5.70)^{* *}$ \\
\hline $0.5 \mathrm{~h}-\mathrm{BG}(\mathrm{mmol} / \mathrm{L})^{\dagger}$ & $7.40(6.49-8.45)$ & $9.06(8.28-9.79)^{* *}$ & $9.64(8.86-10.78)^{* * \Delta}$ \\
\hline $1 \mathrm{~h}-\mathrm{BG}(\mathrm{mmol} / \mathrm{L})^{\dagger}$ & $6.07(4.99-7.20)$ & $8.14(7.17-9.55)^{* *}$ & $10.10(9.38-11.43)^{* *} \boldsymbol{\Lambda}$ \\
\hline $2 \mathrm{~h}-\mathrm{BG}(\mathrm{mmol} / \mathrm{L})^{\dagger}$ & $5.42(4.66-6.25)$ & $6.47(5.97-7.20)^{* *}$ & $8.73(8.10-9.40)^{* * \Delta}$ \\
\hline FIns $(\mathrm{mU} / \mathrm{L})^{\dagger}$ & $7.14(5.94-8.73)$ & $18.42(12.00-24.63)^{* *}$ & $20.39(13.90-27.95)^{* *}$ \\
\hline $0.5 \mathrm{~h}-\mathrm{Ins}(\mathrm{mU} / \mathrm{L})^{\dagger}$ & $81.96(54.38-115.84)$ & $146.50(96.98-250.40)^{* *}$ & $135.85(83.48-198.80)^{* *}$ \\
\hline 1h-Ins $(\mathrm{mU} / \mathrm{L})^{\dagger}$ & $54.64(36.80-83.61)$ & $162.65(115.70-251.48)^{* *}$ & $171.25(121.15-241.35)^{* *}$ \\
\hline $2 \mathrm{~h}-\operatorname{Ins}(\mathrm{mU} / \mathrm{L})^{\dagger}$ & $41.57(24.21-63.11)$ & $118.70(89.73-182.33)^{* *}$ & $197.15(129.58-289.20)^{* * \Delta}$ \\
\hline $\mathrm{AUCi}^{\dagger}$ & $107.5(61.1-139.0)$ & $277.0(188.6-406.2)^{* *}$ & $293.1(206.1-442.2)^{* *}$ \\
\hline AUCg & $11.7(10.3-13.4)$ & $15.0(14.2-16.6)^{* *}$ & $18.4(17.2-19.5)^{* * \Delta}$ \\
\hline HOMA2-IR ${ }^{\dagger}$ & $1.00(0.84-1.27)$ & $2.77(1.76-3.58)^{* *}$ & $2.99(2.06-4.11)^{* *}$ \\
\hline Fetuin-B (mg/L) & $5.38 \pm 3.50$ & $6.89 \pm 3.61^{*}$ & $8.13 \pm 4.86^{* * \Delta}$ \\
\hline M-value ${ }^{\dagger}$ & $10.29(8.63-11.46)$ & $4.32(3.59-5.13)^{* *}$ & $3.98(2.98-5.29)^{* *}$ \\
\hline
\end{tabular}

2 Values are given as mean \pm SD or median (Inter quartile Range). BMI, body mass index; FAT

$3(\%)$, the percentage of fat in body; WC, waist circumference; SBP, systolic blood pressure;

4 DBP, diastolic blood pressure; TG, triglyceride; TC, total cholesterol; HDL-C, high-density 
5 lipoprotein cholesterol; LDL-C, low-density loprotein cholesterol; FFA, free fatty acid; FBG, 6 fasting blood glucose; 0.5h-BG, $0.5 \mathrm{~h}$ blood glucose after glucose overload; 1h-BG, 1-h blood 7 glucose after glucose overload; 2h-BG, 2-h blood glucose after glucose overload; FIns, fasting 8 plasma insulin; 2h-Ins, 2-h plasma insulin after glucose overload; AUCi, the area under the 9 curve for insulin; AUCg, the area under the curve for glucose; HOMA-IR, homeostasis model 10 assessment of insulin resistance; $\quad$, Log transformed before analysis; ${ }^{*} p<0.05,{ }^{* *} p<0.01$ 11 compared with NGT group; ${ }^{\Delta} p<0.05,{ }^{\Delta} p<0.01$ compared with IR-N 


\section{Table 2 (on next page)}

Linear regression analysis of variables associated with serum Fetuin-B levels in young woman 
1

\begin{tabular}{|c|c|c|c|c|c|c|}
\hline \multirow[b]{2}{*}{ Variable } & \multicolumn{2}{|c|}{ Model 1} & \multicolumn{2}{|c|}{ Model 2} & \multicolumn{2}{|c|}{ Model 3} \\
\hline & $\mathrm{r}$ & $\mathrm{p}$ & $\mathrm{r}$ & $\mathrm{p}$ & B & $\mathrm{p}$ \\
\hline Age (years) & 0.042 & 0.473 & & & & \\
\hline FAT (\%) & 0.195 & $<0.01$ & & & & \\
\hline BMI (kg/m2) & 0.242 & $<0.001$ & 0.119 & 0.057 & & \\
\hline $\mathrm{WC}(\mathrm{cm})$ & 0.252 & $<0.001$ & 0.191 & $<0.05$ & & \\
\hline SBP (mmHg) & 0.166 & $<0.01$ & 0.077 & 0.222 & & \\
\hline $\mathrm{DBP}(\mathrm{mmHg})$ & 0.146 & $<0.05$ & 0.102 & 0.106 & & \\
\hline $\mathrm{TC}(\mathrm{mmol} / \mathrm{L})$ & 0.089 & 0.127 & 0.015 & 0.807 & & \\
\hline $\mathrm{TG}(\mathrm{mmol} / \mathrm{L}) \dagger$ & 0.150 & $<0.05$ & 0.056 & 0.374 & & \\
\hline $\mathrm{HDL}-\mathrm{C}(\mathrm{mmol} / \mathrm{L}) \dagger$ & -0.049 & 0.397 & -0.029 & 0.650 & & \\
\hline LDL-C (mmol/L) & 0.088 & 0.130 & 0.009 & 0.887 & & \\
\hline $\mathrm{FFA}(\mu \mathrm{mol} / \mathrm{L}) \dagger$ & -0.038 & 0.515 & -0.030 & 0.636 & & \\
\hline HbA1c $(\%) \dagger$ & 0.201 & $<0.001$ & 0.125 & 0.046 & & \\
\hline FBG $(\mathrm{mmol} / \mathrm{L}) \dagger$ & 0.171 & $<0.01$ & 0.067 & 0.288 & & \\
\hline $2 \mathrm{~h}-\mathrm{BG}(\mathrm{mmol} / \mathrm{L}) \dagger$ & 0.219 & $<0.001$ & 0.108 & 0.087 & & \\
\hline FIns $(\mathrm{mU} / \mathrm{L}) \dagger$ & 0.261 & $<0.001$ & 0.172 & $<0.05$ & & \\
\hline 2h-Ins (mU/L)† & 0.220 & $<0.001$ & 0.095 & 0.132 & & \\
\hline AUCi $\dagger$ & 0.182 & $<0.01$ & 0.063 & 0.315 & & \\
\hline AUCg & 0.155 & $<0.01$ & 0.031 & 0.627 & & \\
\hline HOMA2-IR† & 0.262 & $<0.001$ & 0.170 & $<0.05$ & & \\
\hline M-value $†$ & -0.299 & $<0.001$ & -0.230 & $<0.001$ & -0.379 & $<0.001$ \\
\hline
\end{tabular}

2 Model 1: unadjusted simple linear regression analysis. Model 2: adjusted age and FAT\%,

3 partial linear regression analysis. Model 3: multiple linear stepwise regression analysis,

4 values included for analysis were BMI, WC, SBP, DBP, FAT\%, TG, HOMA2-IR and M-value. †,

5 Log transformed before analysis. 


\section{Table 3 (on next page)}

Main clinical and metabolic features pre- and post-treatment with GLP-1RA in IGT and IR-NG women 


\begin{tabular}{|c|c|c|c|}
\hline Variable & & Post-treatment & Post-treatment \\
\hline & Baseline & 3 months & 6 months \\
\hline $\mathrm{BMI}(\mathrm{kg} / \mathrm{m} 2)$ & $28.43 \pm 3.13$ & $26.44 \pm 2.97^{*}$ & $25.91 \pm 3.38^{* *}$ \\
\hline Fat $(\%)$ & $39.99 \pm 5.54$ & $36.25 \pm 4.12^{*}$ & $35.88 \pm 5.02^{* *}$ \\
\hline $\mathrm{WC}(\mathrm{cm})$ & $88.86 \pm 7.25$ & $85.98 \pm 7.10$ & $84.56 \pm 7.60^{*}$ \\
\hline $\mathrm{SBP}(\mathrm{mmHg})$ & $116.72 \pm 11.72$ & $111.32 \pm 10.23$ & $112.68 \pm 12.33$ \\
\hline DBP (mmHg) & $74.08 \pm 8.53$ & $73.80 \pm 10.08$ & $73.24 \pm 9.24$ \\
\hline $\mathrm{TC}(\mathrm{mmol} / \mathrm{L})$ & $4.74 \pm 0.74$ & $4.26 \pm 0.89 *$ & $4.33 \pm 0.82$ \\
\hline $\mathrm{TG}(\mathrm{mmol} / \mathrm{L})$ & $1.89 \pm 0.64$ & $1.55 \pm 0.67$ & $1.41 \pm 0.68^{*}$ \\
\hline HDL-C (mmol/L) & $1.14 \pm 0.26$ & $1.05 \pm 0.18$ & $1.08 \pm 0.19$ \\
\hline LDL-C (mmol/L) & $3.00 \pm 0.71$ & $2.63 \pm 0.82$ & $2.62 \pm 0.80$ \\
\hline FFAs $(\mu \mathrm{mol} / \mathrm{L})$ & $0.50 \pm 0.17$ & $0.44 \pm 0.12$ & $0.45 \pm 0.22$ \\
\hline HbA1c (\%) & $5.50 \pm 0.36$ & $5.26 \pm 0.26^{* *}$ & $5.20 \pm 0.24^{* *}$ \\
\hline FBG (mmol/L) & $5.37(5.14-5.47)$ & $5.17(5.03-5.46)$ & $5.14(4.93-5.24)^{*}$ \\
\hline $0.5 \mathrm{~h}-\mathrm{BG}(\mathrm{mmol} / \mathrm{L})$ & $9.28(8.60-10.23)$ & $8.66(7.58-10.15)$ & $8.59(7.67-9.42)^{*}$ \\
\hline 1h-BG (mmol/L) & $9.14(7.62-10.48)$ & $8.46(6.74-9.85)$ & $8.38(7.49-9.79)$ \\
\hline 2h-BG (mmol/L) & $7.81(6.82-8.83)$ & $6.84(5.59-7.97)$ & $6.71(5.73-8.29)^{*}$ \\
\hline FIns (mU/L) & $21.69(16.00-31.07)$ & $17.66(11.43-24.13)^{*}$ & $13.42(9.71-22.59)^{* *}$ \\
\hline $0.5 \mathrm{~h}-\mathrm{Ins}(\mathrm{mU} / \mathrm{L})$ & $172.95(126.80-210.10)$ & $145.70(93.27-240.38)$ & $142.05(91.20-209.55)$ \\
\hline 1h-Ins (mU/L) & $173.70(119.30-334.10)$ & $179.35(108.73-261.58)$ & $185.20(121.95-257.08)$ \\
\hline 2h-Ins (mU/L) & $174.70(119.95-269.58)$ & $154.25(78.09-252.93)$ & $121.05(78.76-194.08)$ \\
\hline AUCi & $285.3(224.3-556.5)$ & $304.3(176.4-466.0)$ & $290.8(192.6-399.5)$ \\
\hline AUCg & $16.9(14.9-18.3)$ & $15.5(13.0-17.3)$ & $15.0(13.9-17.4)$ \\
\hline
\end{tabular}


HOMA2-IR

M-value

Fetuin-B (mg/L)
$0.83(0.78-0.85)$

$3.76 \pm 0.73$

$8.08 \pm 1.77$
$0.78(0.76-0.85)$

$5.11 \pm 1.70^{* *}$

$7.47 \pm 1.48$
$0.77(0.73-0.80)^{*}$

$5.27 \pm 1.79^{* *}$

$6.75 \pm 1.57^{*}$

2 Values were given as mean \pm SD or median (Inter quartile Range). ${ }^{*} \mathrm{p}<0.05,{ }^{* *} \mathrm{p}<0.01$ vs.

3 Baseline; $\Delta \mathrm{p}<0.05, \Delta \mathrm{p}<0.01$ vs. post-treatment 3 months. 